\title{
LA CLASE INVERTIDA EN LA EDUCACIÓN SUPERIOR: PERCEPCIONES DEL ALUMNADO
}

\author{
Graciela Arráez Vera \\ Graciela.arraez@ua.es \\ Alejandro Lorenzo Lledó \\ alejandro.lorenzo@ua.es \\ Marcos Gómez Puerta \\ marcos.gomez@ua.es \\ Gonzalo Lorenzo Lledó \\ glledo@ua.es \\ Universidad de Alicante \\ Facultad de Educación
}

Fecha de Recepción: 16 Marzo 2018

Fecha de Admisión: 10 Abril 2018

\section{RESUMEN}

El aula invertida es una metodología pedagógica innovadora. Son múltiples las habilidades que este enfoque aporta al estudiante durante el proceso de enseñanza-aprendizaje. En la asignatura del segundo curso del Grado de Maestro/a de Educación Primaria denominada Atención a las Necesidades Educativas Específicas (ANEE) se ha implementado esta metodología. El objetivo de este trabajo consistió en evaluar y describir las percepciones que los estudiantes tienen después de haber cursado una asignatura a través de esta metodología. Para lograr la consecución de dicho objetivo se utilizó el cuestionario de percepciones de los estudiantes sobre la clase invertida (Gilboy, Heinerichs y Pazzaglia, 2015). Este cuestionario consiste en una escala tipo Likert y evalúa el grado de satisfacción de los estudiantes con respecto a esta metodología. Unas semanas antes de la finalización de la asignatura, se administró al alumnado del grupo de la asignatura de ANEE. La muestra estuvo conformada por 45 alumnos y alumnas. Los resultados mostraron que el $68,8 \%$ de los estudiantes prefieren la clase invertida en vez de las clases tradicionales y el $77,7 \%$ considera que asimiló mejor los contenidos con la clase invertida que con la metodología tradicional.

Palabras clave: clase invertida; enseñanza-aprendizaje; educación superior; percepciones alumnado

\section{ABSTRACT \\ "The flipped classroom in higher education: student perceptions".}




\section{LA CLASE INVERTIDA EN LA EDUCACIÓN SUPERIOR: PERCEPCIONES DEL ALUMNADO}

The Flipped Classroom is an innovative pedagogical methodology. This approach brings multiple skills to the student during the teaching-learning process. In the subject of the second year of the Primary Education Teacher's Degree, Attention to Specific Educational Needs (ASEN) this methodology has been implemented. The objective of this work was to evaluate and describe the perceptions that students have after having completed a subject through this methodology. In order to achieve this goal, the questionnaire of students' perceptions about the Flipped Classroom was used (Gilboy, Heinerichs \& Pazzaglia, 2015). This questionnaire consists of a Likert-type scale and evaluates the degree of student satisfaction with respect to this methodology. A few weeks before the end of the subject, it was administered to the students of the group of the subject of ASEN. The sample consisted of 45 students. The results showed that $68,8 \%$ of the students prefer the Flipped Classroom instead of the traditional classes and $77,7 \% 77.7 \%$ of the students consider that they assimilated the contents better with the Flipped Classroom than with the traditional methodology.

Keywords: flipped classroom; teaching-learning; higher education; student perceptions

\section{ANTECEDENTES}

La revolución tecnológica producida durante la última década ha promovido la irrupción de nuevas metodologías. Son múltiples las ventajas que se le otorgan a las metodologías que incluyen el uso de las tecnologías frente a la metodología tradicional. Entre ellas se destacan: una mayor atención sostenida (Bunce, Flens y Neiles, 2010), el desarrollo de un pensamiento crítico, una mejora sustancial tanto en la comunicación escrita como en las habilidades de razonamiento (Arum y Roska, 2010) y un incremento de la integración de los conocimientos. Otros autores (Kong, 2014; McLaughlin et al., 2014) revelan que además esta metodología mejora la gestión del conocimiento del alumnado, ya que los materiales facilitados por el profesor les ayudan a preparar cada sesión de clase y por tanto les ayuda a mantener un papel más activo en el proceso de enseñanza- aprendizaje el cual resulta clave para alcanzar una mayor tasa de retención en los contenidos.

La metodología Flipped Classroom o clase invertida experimentó un gran auge cuando en 2006 los profesores Bergmann y Sams (2006) comenzaron a distribuir entre su alumnado videos de sus lecciones como apoyo complementario a sus explicaciones. De este modo, descubrieron que además de facilitar la comprensión de los contenidos de la materia disponían de más tiempo en clase para resolver dudas o cuestiones prácticas. Sucintamente, la clase invertida consistiría en invertir la manera en la que el docente presenta los contenidos con la finalidad de conseguir mayor tiempo para la práctica y para la aplicación de teorías y conceptos (Talbert, 2012). La implementación de esta metodología compromete a los estudiantes a revisar los contenidos teóricos fuera del aula y la práctica se realiza durante el transcurso de la clase. Con este enfoque se persigue que el estudiante sea una pieza activa implicada en el proceso enseñanza-aprendizaje, donde tenga primacía el aprendizaje natural frente al aprendizaje tradicional (García Cué, Santizo Rincón y Alonso García 2009).

Esta metodología está en consonancia con los principios que promulga el Espacio Europeo de Educación Superior que exige no sólo conseguir las competencias prácticas adecuadas a la materia, sino adquirir conocimientos teóricos a través de actividades prácticas. No obstante, se ha de tener en cuenta que la implementación de este enfoque exige un compromiso por parte del docente y del discente, ya que el docente debe preparar con antelación gran cantidad de material y facilitar a los estudiantes los materiales necesarios antes del inicio de la clase. Por otra parte, los alumnos y alumnas han de comprometerse y trabajar con asiduidad, pues todo el material facilitado por parte del profesor debe ser consultado con antelación antes de cada clase. Además, el aprendizaje activo por parte del estudiante implicará, en algunas ocasiones, que sea el propio discente el que tenga que buscar información relacionada, modificarla o incluso crearla. 
En los últimos años, el enfoque del aula invertida se ha vuelto cada vez más popular (Lo, Lie y Hew, 2018), pues las conclusiones encontradas en la literatura científica parecen avalar los buenos resultados que se consiguen tras implementar esta metodología (Betihavas, Bridgman, Kornhaber, y Cross, 2016; O'Flaherty y Phillips, 2015; Tune, Sturek y Basile, 2013). Ejemplo de ello es el estudio realizado por Pierce y Fox (2012) donde compararon los rendimientos académicos de los estudiantes que acudieron a la clase en la cual se implementó una clase invertida frente a los que acudieron a una clase con la metodología tradicional. Los estudiantes en la clase invertida tuvieron rendimientos académicos significativamente más altos que aquellos que acudieron a la clase tradicional. Asimismo, los estudiantes tuvieron opiniones positivas sobre las estrategias de aprendizaje activo.

En cuanto a la educación superior, otra de las ventajas que respalda la utilización de este enfoque es el hecho de que en la educación superior el tiempo de docencia resulta a veces muy limitado. En estos casos, la metodología del aula invertida ofrece muchas ventajas para poder trabajar todos los contenidos de la materia, ya que este enfoque instructivo permite un uso más efectivo del tiempo dentro del aula. Los maestros están disponibles todo el tiempo en el aula consiguiéndose así una retroalimentación inmediata (O'Flaherty y Phillips, 2015), pues al entregar el material y permitir que los alumnos y alumnas visualicen los contenidos fuera de la clase se consigue que se adquieran algunos conocimientos básicos antes de clase y dedicar más tiempo en el aula a la realización de ejercicios activos de aprendizaje tales como la resolución colaborativa de problemas, discusiones en equipo o presentaciones de estudiantes (Abeysekera y Dawson, 2015; Roehl, Reddy y Shannon, 2013) que pueden llevarse a cabo con la orientación de un profesor.

\section{OBJETIVOS}

El objetivo general de este estudio ha sido implementar la metodología del aula invertida en la asignatura de ANEE del Grado de Magisterio de Educación Primaria de la Universidad de Alicante y evaluar el grado de satisfacción de los estudiantes sobre la metodología empleada. Como objetivos específicos se destacan:

Identificar con qué metodología, clase invertida frente a clase tradicional, el alumnado considera que asimila mejor los contenidos.

Analizar las ventajas y los inconvenientes que los estudiantes perciben con la implementación de esta metodología.

Identificar el tipo de materiales y actividades que el alumnado considera más útiles para una mejor asimilación de contenidos.

\section{PARTICIPANTES}

La experiencia docente se desarrolló durante el segundo cuatrimestre del curso académico 2017-2018 en la asignatura ANEE perteneciente al segundo curso de la titulación del Grado de Magisterio de Educación Primaria de la Universidad de Alicante. En esta propuesta participaron 45 alumnos de un total de 51 (88\%), de los cuales 13 (28.9\%) eran hombres y $32(71.1 \%)$ eran mujeres.

\section{METODOLOGíA}

Para poder llevar a cabo este estudio, en primer lugar, se valoró la posibilidad de modificar las clases tradicionales en clases invertidas para la asignatura ANEE. El primer día de clase se planteó a los estudiantes la posibilidad de utilizar esta nueva metodología frente a la clase tradicional. Se les explicó cómo sería la dinámica de las clases y se informó que sería necesario en casa un tiempo de dedicación para revisar el material. La totalidad de la clase estuvo de acuerdo en emplear esta metodología y se comprometieron a trabajar en casa. 


\section{LA CLASE INVERTIDA EN LA EDUCACIÓN SUPERIOR: PERCEPCIONES DEL ALUMNADO}

El segundo paso, fue facilitar a los alumnos y las alumnas la primera semana de clase todo el material necesario para trabajar en casa. Este material consistía en un dossier con todos los temas de la asignatura. Se emplearon también cinco vídeos alojados en YouTube en los cuales se recogía información complementaria que permitían una mejor comprensión de los contenidos del temario.

En cuanto a la metodología empleada durante las clases se realizaron actividades diversas. En la asignatura de ANEE las clases son dos veces a la semana. El primer día de la semana se comenzaba con una evaluación formativa del tema que previamente había sido leído en casa por el estudiante. A través de un guion de preguntas elaborado por la profesora se iniciaba una discusión de los contenidos mediante preguntas y respuestas. En algunas ocasiones se realizaba una breve exposición por parte de la profesora y se aclaraban las posibles dudas que habían surgido tras la lectura del tema. El segundo día de la semana de clase se dedicaba a realizar trabajo activo en grupos de cuatro integrantes, que contemplaban resolución de casos prácticos y discusión en grupo. Los estudiantes accedían a información mediante smartphone, PC y/o dossier de la asignatura. Una vez finalizada la clase se sugería a los estudiantes que profundizaran sobre los temas tratados durante la clase y los complementaran con información adicional (videos alojados en YouTube). En la resolución de los casos prácticos elaborados por los estudiantes se realizaba la retroalimentación y calificación formativa del mismo.

La recogida de datos se llevó a cabo tras 10 semanas de la implementación de esta metodología. Se distribuyó de forma online un cuestionario. El cuestionario empelado en este estudio para evaluar sus percepciones sobre el entorno de aprendizaje invertido en el aula fue diseñado por Gilboy et al., (2015). Este cuestionario consiste en una escala Likert con cinco ítems en los cuales las opciones de respuestas eran: muy de acuerdo, de acuerdo, neutral, en desacuerdo o muy en desacuerdo. Este cuestionario presenta un Alfa de Cronbach con un valor de .71, un valor aceptable para la confiabilidad. El cuestionario también incluyó dos preguntas abiertas sobre las opiniones de los estudiantes sobre el trabajo antes y durante la clase invertida. De un total de 51 estudiantes, 45 $(88 \%)$ completaron voluntariamente el cuestionario.

\section{RESULTADOS}

Los resultados obtenidos se presentan en la tabla 2. Para ello, se ha realizado un análisis descriptivo de las frecuencias, porcentajes, medias y desviaciones típicas de las puntuaciones obtenidas por el alumnado tras la administración del cuestionario. 
Tabla 1

Análisis descriptivo del grado de satisfacción con la metodología del aula invertida

\begin{tabular}{|c|c|c|c|c|c|c|c|c|c|c|c|c|c|}
\hline \multirow[t]{2}{*}{ Ítems } & \multirow[t]{2}{*}{$\mathrm{n}$} & \multicolumn{2}{|c|}{$\begin{array}{l}\text { Muy en } \\
\text { desacuer } \\
\text { do }(\%)\end{array}$} & \multicolumn{2}{|c|}{$\begin{array}{c}\text { En } \\
\text { desacuerd } \\
\text { o }(\%) \\
\end{array}$} & \multicolumn{2}{|c|}{ Neutral } & \multicolumn{2}{|c|}{$\begin{array}{c}\text { De } \\
\text { acuerdo }\end{array}$} & \multicolumn{2}{|c|}{$\begin{array}{l}\text { Muy de } \\
\text { acuerdo }\end{array}$} & \multirow[b]{2}{*}{ M } & \multirow[b]{2}{*}{ DE } \\
\hline & & $\sum$ & $\%$ & $\sum$ & $\%$ & $\Sigma$ & $\%$ & $\sum$ & $\%$ & $\sum$ & $\%$ & & \\
\hline $\begin{array}{l}\text { 1. Me gustó la posibilidad } \\
\text { de ver un vídeo en vez de } \\
\text { tener una clase tradicional } \\
\text { de los temas del curso }\end{array}$ & 45 & & & 6 & 13,3 & 4 & 8,8 & 14 & 31,1 & 21 & 46,6 & 4,1 & 1 \\
\hline $\begin{array}{l}\text { 2. Prefiero la clase } \\
\text { tradicional del profesor } \\
\text { en vez de realizar trabajos } \\
\text { activos y grupales en } \\
\text { clases como los que se } \\
\text { llevaron a cabo con } \\
\text { metodología del aula } \\
\text { invertida }\end{array}$ & 45 & 15 & 33,3 & 16 & 35,5 & 9 & 20 & 5 & 11,1 & & & 2 & 0,9 \\
\hline $\begin{array}{l}\text { 3. El uso de vídeos me } \\
\text { permite aprender el } \\
\text { material de estudio más } \\
\text { eficazmente que hacer las } \\
\text { lecturas en solitario. }\end{array}$ & 45 & & & 6 & 13,3 & & & 19 & 42,2 & 20 & 44,4 & 4,1 & 0,9 \\
\hline 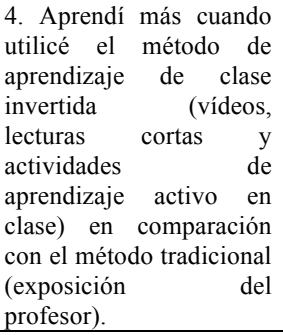 & 45 & & & 7 & 15,5 & 3 & 6,6 & 17 & 37,7 & 18 & 40 & 4 & 1 \\
\hline $\begin{array}{l}5 . \quad \text { Me sentía } \\
\text { desconectado sin un } \\
\text { profesor presente durante } \\
\text { los vídeos o actividades } \\
\text { virtuales. }\end{array}$ & 45 & & & 6 & 13,3 & 9 & 20 & 13 & 28,8 & 17 & 37,7 & 3,9 & 1 \\
\hline
\end{tabular}

Los valores obtenidos en la tabla 2 demuestran que un $77,7 \%$ de los estudiantes prefiere el visionado de videos en vez de tener una clase tradicional. La mayoría de los estudiantes, un 68,8\% prefiere participar en clase a través de la realización de trabajos activos y grupales que una clase tradicional. En relación con el material empleado, un $86,6 \%$ del alumnado considera que aprendió más a través del visionado de videos que haciendo lecturas en solitario. En cuanto a la asimilación de contenidos, un $77,7 \%$ considera que aprendió más con el método de aprendizaje de la clase invertida en comparación con el método tradicional. Por último, un $66,5 \%$ de los alumnos y alumnas sugieren que se sentían desconectados sin un profesor que les guíe cuando consultan el material en casa.

Por otra parte, los comentarios abiertos del cuestionario revelaron que a los estudiantes les gustó la capacidad de trabajar a su propio ritmo y tiempo (22,2\%). Las preocupaciones planteadas 


\section{LA CLASE INVERTIDA EN LA EDUCACIÓN SUPERIOR: PERCEPCIONES DEL ALUMNADO}

por los estudiantes incluyen no tener al profesor disponible para hacer preguntas durante la parte fuera de clase (31\%), también se cuestiona la posibilidad de que otros estudiantes no estén preparados para emplear las estrategias de aprendizaje activo en las actividades grupales que se realizan en clase $(26 \%)$ y un $40 \%$ del alumnado reconoce que no siempre dedica tiempo a revisar el material en casa.

\section{DISCUSIÓN Y CONCLUSIONES}

El objetivo general de este estudio ha sido analizar el grado de satisfacción que los estudiantes universitarios presentan ante la metodología de la clase invertida. En relación con esta cuestión, nuestros datos están en consonancia con los obtenidos por (Faundez, Bastias y Polanco, 2016; Gilboy et al., 2015; Mok, 2014), puesto que un porcentaje muy alto de los alumnos y alumnas $(77,7 \%)$ prefiere la clase invertida a la clase tradicional.

En cuanto a los objetivos específicos del este trabajo se han extraído varias conclusiones. Por una parte, la presencia del docente parece resultar indispensable para a la asimilación de los contenidos, ya que cuando el alumno/a se enfrenta al aprendizaje en solitario se siente desconectado. Aun así, el estudiante sigue prefiriendo esta metodología frente a la clase tradicional. Otro de los objetivos que se perseguían con este estudio era analizar el tipo de materiales con el que el estudiante percibe que asimila mejor los contenidos. Los resultados muestran una clara preferencia $(86,6 \%)$ del visionado de videos frente a la lectura de textos, artículos y/o dossier de la asignatura.

Estos resultados sugieren ciertas implicaciones educativas. Por un lado, proporcionar el material preferido por el alumno/a requiere mucho tiempo inicial por parte del profesor/a para digitalizar conferencias y pensar en estrategias apropiadas de aprendizaje activo para usar en clase. Otro dato que llama la atención es el alto porcentaje de alumnos/as (40\%) que reconocen no dedicar tiempo suficiente a revisar el material proporcionado por el profesor/a en casa. Por este motivo y en consonancia con diversos autores (Gilboy et al., 2015; Coates, 2006) resulta importante obtener la aprobación de los estudiantes el primer día de clase para la utilización de esta metodología. Sin embargo, en este estudio, aunque el alumnado estuvo de acuerdo y se comprometió con esta metodología el primer día de clase, no se han conseguido buenos resultados. La razón de esta falta de compromiso por parte de los estudiantes puede estar relacionada, tal y como apuntan Flaherty y Philips (2015), al tipo de material utilizado, pues según estos autores es menos probable que los estudiantes participen en actividades previas a la clase que carecen de interactividad, ya que no proporcionan mecanismos de retroalimentación formativa y no están vinculados coherentemente con la clase presencial.

A pesar de las aportaciones de este trabajo, cabe señalar la existencia de algunas limitaciones. Una de ellas es que los resultados obtenidos se han centrado únicamente en la satisfacción de los alumnos con respecto a su experiencia con la metodología de la clase invertida. Sin embargo, hubiera sido aconsejable no sólo valorar las percepciones de los estudiantes, sino también evaluar los rendimientos académicos conseguidos tras la implementación de esta metodología.

\section{REFERENCIAS BIBLIOGRÁFICAS}

Abeysekera, L. y Dawson, P. (2015). Motivation and cognitive load in the flipped classroom: Definition, rationale and a call for research. Higher Education Research \& Development, 34(1), 1-14.

Arum, R. y Roksa, J. (2011). Academically adrift: Limited learning on college campuses University of Chicago Press.

Bergmann, J. y Sams, A. (2012). Flip your classroom: Reach every student in every class every day International Society for Technology in Education. 
Betihavas, V., Bridgman, H., Kornhaber, R. y Cross, M. (2016). The evidence for 'flipping out': A systematic review of the flipped classroom in nursing education. Nurse Education Today, 38, 15-21.

Bunce, D. M., Flens, E. A. y Neiles, K. Y. (2010). How long can students pay attention in class? A study of student attention decline using clickers. Journal of Chemical Education, 87(12), 14381443.

Coates, H. (2006). Student engagement in campus-based and online education: University connections Routledge.

Cué, J. L. G., Rincón, J. A. S. y García, C. M. A. (2009). Uso de las TIC de acuerdo a los estilos de aprendizaje de docentes y discentes. Revista Iberoaméricana De Educación, 48(2), 2.

Faundez, A. R. 0., Bastias, J. M. A. y Polanco, M. P. R. (2016). Evaluación de metodología flipped classroom: Primera experiencia. Innoeduca.International Journal of Technology and Educational Innovation, 2(2), 90-99.

Furse, C. (2013). A busy professor's guide to sanely flipping your classroom. Paper presented at the Antennas and Propagation Society International Symposium (APSURSI), 2013 IEEE, 21712172.

Gilboy, M. B., Heinerichs, S. y Pazzaglia, G. (2015). Enhancing student engagement using the flipped classroom. Journal of Nutrition Education and Behavior, 47(1), 109-114. doi: 10.1016/j.jneb.2014.08.008 [doi]

Kong, S. C. (2014). Developing information literacy and critical thinking skills through domain knowledge learning in digital classrooms: An experience of practicing flipped classroom strategy. Computers \& Education, 78, 160-173.

Lewis, C. E., Chen, D. C. y Relan, A. (2018). Implementation of a flipped classroom approach to promote active learning in the third-year surgery clerkship. American Journal of Surgery, 215(2), 298-303. doi: S0002-9610(17)30687-6 [pii]

Lo, C. K., Lie, C. W. y Hew, K. F. (2018). Applying "First principles of instruction" as a design theory of the flipped classroom: Findings from a collective study of four secondary school subjects. Computers \& Education, 118, 150-165.

McLaughlin, J. E., Roth, M. T., Glatt, D. M., Gharkholonarehe, N., Davidson, C. A., Griffin, L. M., . . Mumper, R. J. (2014). The flipped classroom: A course redesign to foster learning and engagement in a health professions school. Academic Medicine: Journal of the Association of American Medical Colleges, 89(2), 236-243. doi:10.1097/ACM.0000000000000086 [doi]

Mok, H. N. (2014). Teaching tip: The flipped classroom. Journal of Information Systems Education, 25(1), 7.

O'Flaherty, J. y Phillips, C. (2015). The use of flipped classrooms in higher education: A scoping review. The Internet and Higher Education, 25, 85-95.

Pierce, R. y Fox, J. (2012). Vodcasts and active-learning exercises in a "flipped classroom" model of a renal pharmacotherapy module. American Journal of Pharmaceutical Education, 76(10), 196.

Roehl, A., Reddy, S. L. y Shannon, G. J. (2013). The flipped classroom: An opportunity to engage millennial students through active learning. Journal of Family and Consumer Sciences, 105(2), 44.

Sams, A. y Bergmann, J. (2013). Flip your students' learning. Educational Leadership, 70(6), 16-20. Talbert, R. (2012). Inverted classroom. Colleagues, 9(1), 7.

Tune, J. D., Sturek, M. y Basile, D. P. (2013). Flipped classroom model improves graduate student performance in cardiovascular, respiratory, and renal physiology. Advances in Physiology Education, 37(4), 316-320. 


\section{LA CLASE INVERTIDA EN LA EDUCACIÓN SUPERIOR: PERCEPCIONES DEL ALUMNADO}

Wilson, S. G. (2013). The flipped class: A method to address the challenges of an undergraduate statistics course. Teaching of Psychology, 40(3), 193-199. 